\title{
Inklusive Solidarität als Antwort der Gewerkschaften auf den Rechtspopulismus
}

\author{
THOMAS FISCHER
}

\section{Der Nährboden des Rechtspopulismus}

Die Angst vor sozialem Abstieg und Statusverlust und Zukunftssorgen für sich und die eigenen Kinder sind unter AfD-Wählerinnen und -Wählern weit verbreitet. Viele von ihnen sehen sich persönlich zurückgesetzt, ordnen sich - unabhängig von ihrem realen Einkommen - in der Gesellschaft niedrig ein. Sie haben das Gefühl, zu kurz zu kommen - ein Gefühl, das nicht zuletzt die besonders hohen Wahlergebnisse der AfD in den ostdeutschen Bundesländern erklärt. Zugleich verbindet die Wählerschaft der AfD das Empfinden eines dreifachen Kontrollverlusts: Viele von ihnen fühlen sich im Arbeitsleben fremdbestimmt. Politisch sehen sie ihre Interessen durch die etablierten demokratischen Parteien nicht mehr vertreten. Offene Grenzen sind für sie - gerade vor dem Hintergrund der Flüchtlingsfrage - der Beleg, dass dem Nationalstaat in einer globalisierten Welt komplett die Kontrolle entglitten ist. ${ }^{1}$

Natürlich treffen die Rechtspopulisten besonders den Ton von Menschen mit rechtsextremen, autoritären, rassistischen und völkisch-nationalistischen Einstellungen, die sich auch in der Arbeiterschaft finden. Dass sich (männliche) Arbeiter überdurchschnittlich häufig für die AfD entscheiden, hat jedoch andere Gründe. Viele Beschäftigte wählen sie aus Protest gegen die „Zuspitzung arbeitsweltlicher Problemlagen“, die sie täglich im Betrieb erleben. ${ }^{2}$ Insbesondere für die Empfänglichkeit von Gewerkschaftsmitgliedern gegenüber rechtspopulistischem Parolen gibt es eine weitere Erklärung. Viele von ihnen sind frustriert, denn sie werten „Sozialabbau und soziale Spaltung, Abbau von Arbeitnehmer ${ }^{\star}$ innenrechten und ,concession bargaining im Betrieb“ als „Zeugnisse für die nachhaltige Erosion gewerkschaftlicher Schutzfunktionen. [...] Für nicht wenige mündet [dieser Frust] in die - zunächst passive - Hinwendung zu rechten Ideologien.“ ${ }^{3}$

\section{Das trügerische Identitätsversprechen der Rechtspopulisten: \\ Exklusive Solidarität}

Auch aus diesem geschwächten Vertrauen der Beschäftigten in die Durchsetzungsfähigkeit der Gewerkschaften versuchen die Rechtspopulisten Kapital zu schlagen. Sie inszenieren sich sozialpopulistisch als „Arbeiterbewegung von rechts" und instrumentalisieren die Empörung über soziale und ökonomische Ungerechtigkeitsverhältnisse, um so für ihre völkisch-nationalistischen Politikansätze zu mobilisieren.

Die Rechtspopulisten bedienen sich dafür eines kollektiven Identitätsversprechens, das auf exklusiver Solidarität, der Ausgrenzung von Fremden und Abschottung gegen außen beruht. Indem sie die „nationale“ mit der „sozialen Frage" verbinden, münzen die Rechtspopulisten die Auseinandersetzung auf der gesellschaftlichen Oben-Unten-Konfliktachse zwischen Kapital und Arbeit um auf die vermeintliche Innen-Außen-Konfliktachse zwischen „wir Deutsche" und „die Fremden“. Gleichzeitig besetzen die Rechtspopulisten die Oben-Unten-Konfliktachse neu. Verkleidet in den Anspruch, nur die AfD würde die Interessen der "kleinen Leute“ gegen „das verkommene politische Establishment da oben" verteidigen, sagen sie unserer Demokratie den Kampf an.

\section{Drei Dimensionen inklusiver Solidarität für gewerkschaftliches Handeln}

Dieses ideologische Rüstzeug der Rechtspopulisten spricht in Wahrheit allen Ideen und Zielen der Arbeiterbewegung Hohn. Die von den Rechtspopulisten propagierte Innen-Außen-Konfliktachse zielt darauf ab, Belegschaften entlang ethnischer Zugehörigkeiten zu spalten, die Solidarität der Stärkeren mit den Schwächeren zu untergraben und Beschäftigte gegeneinander auszuspielen.

Es bleibt dabei, dass die AfD programmatisch für starke neoliberale, arbeitnehmerinnenund arbeitnehmerfeindliche Positionen steht und an der wachsenden Ungleichverteilung von Einkommen, Vermögen und Lebenschancen in unserer Gesellschaft nicht ernsthaft rütteln will. Dennoch finden ihre Parolen gerade bei der Arbeiterschaft und bei Gewerkschaftsmitgliedern überdurchschnittlich starken Anklang. Für die Gewerkschaften gibt es verschiedene Ansatzpunkte, um den Rechtspopulisten in Arbeitswelt und Betrieb das Wasser abzugraben. Hierzu wird im Folgenden zwischen drei Dimensionen inklusiver Solidarität unterschieden.

Der Kampf gegen Menschenfeindlichkeit, Rassismus und soziale Ausgrenzung: Von Anfang war es das Ziel der gewerkschaftlichen Organisation von inklusiver Solidarität, allen abhängig Beschäftigen ein freies und selbstbestimmtes Leben zu ermöglichen. Heute reicht es aber immer weniger, dabei nur die Arbeits- und sozialen Lebensverhältnisse in den Blick zu nehmen. Vielmehr sind die Gewerkschaften stärker denn je gefordert, ihre Arbeit an einem ganzheitlichen Emanzipationsbegriff auszurichten, der
1 Vgl. Hilmer, R./Kohlrausch, B./Müller-Hilmer, R./ Gagné, J. (2017): Einstellung und soziale Lebenslagen. Eine Spurensuche nach den Gründen für rechtspopulistische Orientierung, auch unter Gewerkschaftsmitgliedern: Hans-Böckler-Stif- tung, Working Paper Forschungsförderung Nr. 44, Düsseldorf.

2 Vgl. Sauer, D./Stöger, U./Bischoff, J./Detje, R./ Müller, B. (2018): Rechtspopulismus und Ge- werkschaften. Eine arbeitsweltliche Spurensuche, Hamburg, S. 22; vgl. auch den Beitrag von Sauer/Detje in diesem Heft.

3 Sauer et al. (2018), a. a. O., S. 22 
„die gesamte Lebensweise der Menschen in den Blick [nimmt], die Seite der Produktion und die der Reproduktion, die der materiellen Bedürfnisse und die Bedürfnisse nach sozialer Anerkennung selbst gewählter Identitäten und Lebensformen. Die Überwindung von Geschlechterhierarchien und rassistischer Diskriminierung, der Anspruch, sexuelle Orientierung ohne Diskriminierung ausleben zu können, sind Bedürfnisse, die zu einer emanzipierten Lebensweise gehören [...]. Es muss [den Gewerkschaften besser] gelingen, Klassenfragen mit [solchen] Anerkennungsund Identitätsfragen zu verbinden." 4

Das bedeutet auch, dass die Gewerkschaften „klare Kante“ zeigen müssen gegenüber jeder Art von Menschenfeindlichkeit, Rassismus und sozialer Ausgrenzung aufgrund von Herkunft, Geschlecht, sexueller Orientierung, Religionszugehörigkeit oder was auch immer - auch auf die Gefahr hin, dass diese konsequente Haltung in den eigenen Reihen nicht nur auf Beifall stößt.

Solidarisches Zusammenstehen auch jenseits des eigenen Organisationsbereichs: Gerade bei ihren Mitgliedern ist das Empfinden schwindender Machtressourcen der Gewerkschaften ein wichtiger Grund für die Anfälligkeit gegenüber rechtspopulistischer Ansprache. Diese Beobachtung gilt, wenn auch in verschiedenen Ausmaßen, für alle „drei Welten der Arbeitsbeziehungen“, wie sie Wolfgang Schroeder beschreibt: ${ }^{5}$

Die erste Welt der Arbeitsbeziehungen setzt sich vornehmlich aus Großbetrieben der verarbeitenden Industrie im exportorientierten Sektor sowie den Kernbereichen des öffentlichen Dienstes zusammen. Gewerkschaften und Arbeitgeberverbände sind nach wie vor einflussreiche Akteure. Sie verfügen über einen vergleichsweise hohen Organisationsgrad. Deshalb ist auch die Tarifbindung in der Fläche noch relativ hoch und die betrieblichen Mitbestimmungsstrukturen sind verhältnismäßig intakt.

In der zweiten Welt der Arbeitsbeziehungen mit ihren mittelgroßen Betrieben und Teilbereichen des öffentlichen Dienstes treten Berufsgewerkschaften in den tarifpolitischen Überbietungswettbewerb mit den DGB-Gewerkschaften und tragen damit zur Spaltung der Belegschaften bei. Flächentarifverträge sind die
Ausnahme, während der Stellenwert von Haustarifverträgen zunimmt.

Die dritte Welt der Arbeitsbeziehungen besteht vor allem aus kleinen und mittelständischen Unternehmen, vielfach in Ostdeutschland, dem einfachen Dienstleistungssektor und dem Handwerk. Der Organisationsgrad von Gewerkschaften und Arbeitgeberverbänden ist gering, die Tarifbindung und betrieblichen Mitbestimmungsstrukturen in der Fläche sind entsprechend schwach und prekäre Beschäftigung ist weit verbreitet.

Allerdings haben sich diese drei Welten zunehmend entkoppelt. Die Löhne, die Arbeitsbedingungen, die Mitbestimmung und die Kooperations- und Konfliktdimensionen zwischen den Sozialpartnern driften immer weiter auseinander. Gleichzeitig „kontaminieren“ die schlechteren Bedingungen in der zweiten und dritten Welt zunehmend die erste Welt und erzeugen auch dort wachsende Unsicherheit für die Beschäftigten - eine Unsicherheit, die angesichts des massiven Strukturwandels durch Globalisierung, Digitalisierung und den Übergang in eine $\mathrm{CO}_{2}$-freie Wirtschaft in allen drei Welten der Arbeitsbeziehungen massiv verstärkt wird.

Um diese "negativen Spillover-Effekte“ der zweiten und dritten Welt auf die erste Welt einzudämmen, muss über alle drei Welten hinweg die Tarifbindung in der Fläche gestärkt werden. Es ist aber längst nicht allen Gewerkschaften möglich, dies aus eigener Kraft durch den Ausbau ihrer Mitgliederstärke und Organisationsmacht durchzusetzen. Zudem unterlaufen die Arbeitgeber durch Tarifflucht und die Möglichkeit von Verbandsmitgliedschaften ohne Tarifbindung („OT-Mitgliedschaft") jeden Versuch in diese Richtung. Deshalb ist es höchste Zeit, dass der Gesetzgeber flankiert, etwa indem er die Allgemeinverbindlicherklärung von Tarifverträgen erleichtert, ihre Nachwirkung stärkt oder bundesweit geltende Tariftreueregelungen einführt. Gleichzeitig ist es primär Aufgabe des Gesetzgebers, die Ordnung auf dem Arbeitsmarkt in allen drei Welten wiederherzustellen. Er muss endlich dafür sorgen, dass der Niedriglohnsektor eingedämmt und der Sumpf prekärer
Beschäftigungsverhältnisse trockengelegt wird.

All dies sind Ziele, für die sich der DGB und seine Mitgliedsgewerkschaften mit vereinter Kraft stark machen. Inklusive Solidarität im Sinne des gewerkschaftlichen Zusammenstehens über die jeweils eigenen Branchen- und Organisationsgrenzen hinaus hat aber noch weitere wichtige Facetten. Wenn es beispielsweise darum geht, die miserablen Beschäftigungsbedingungen prekarisierter Dienstleister*innen im Bereich der Sorgearbeit zu verbessern, ist gerade auch das Engagement der starken Industriegewerkschaften gefragt.

Entsprechende Kooperationsformen zwischen den Gewerkschaften sind sicherlich noch ausbaufähig. ${ }^{6}$ Aber auch hier hat sich schon Einiges bewegt, wie etwa die gemeinsamen Solidaritätsaktionen der DGB-Gewerkschaften in den Arbeitskämpfen zur Aufwertung der Sozial- und Erziehungsberufe zeigen, die wir 2015 organisiert haben.

Engagement für Gute Arbeit und ein gutes Leben: Die dritte Dimension inklusiver Solidarität, die die Gewerkschaften verstärkt in den Blick nehmen, um soziale Haltepunkte für die Beschäftigten zu schaffen und sie so gegen rechtspopulistische Parolen zu immunisieren, betrifft ihr gesellschaftspolitisches Mandat.

Kein Zweifel: Der Betrieb und die Dienststelle bleiben der zentrale Ort gewerkschaftlichen Handelns. Dort sorgen sie mit Tarifvertrag und Mitbestimmung für Gute Arbeit und dafür, dass es gerecht zugeht. Gleichzeitig bergen aber auch die herrschenden Lebensverhältnisse außerhalb der Arbeitswelt Quellen von Verunsicherung und Unzufriedenheit, die den Rechtspopulisten in die Hände spielen können. Angesprochen sind damit z. B. die Sorge um bezahlbares Wohnen oder um eine gute Rente im Alter, fehlende soziale Absicherung, eine unzureichende Versorgung im Gesundheits- und Pflegebereich oder auch ungleiche Bildungschancen. ${ }^{7}$

Auch um diese lebensweltlichen Bezüge, die die Alltagswahrnehmung von Beschäftigten prägen, wollen sich der DGB und seine Mitgliedsgewerkschaften wieder verstärkt kümmern. Deshalb hat der Ordentliche Bundeskongress des DGB im Mai 2018 den DGB-Bundes-
4 Urban, H.-J. (2018): Sind Gewerkschaften (noch Klassenorganisationen?, in: Z. Zeitschrift Marxistische Erneuerung Nr. 116, Dezember 2018, S. 81-95, hier S. 87.

5 Vgl. Schroeder, W. (2016): Konfliktpartnerschaft - still alive. Veränderter Konfliktmodus in der verarbeitenden Industrie, in: Industrielle Beziehungen 23 (3), S. 374-392.

6 Vgl. Emunds, B.: Stirbt die Solidarität als Grundlage gewerkschaftlichen Handelns aus? Solidarität: Wo kommen wir her? Wo sollten wir hin? Impuls beim IG Metall Zukunftsforum am 19. Juni 2018 in Berlin.

7 Vgl. Sauer et al. (2018), a.a.o. (Fußn. 2), S. 149-151, und Hilmer et al. (2017), a.a. O. (Fußn. 1), S. 32 
vorstand damit beauftragt, einen breit angelegten Zukunftsdialog zu initiieren. Wir werden diesen Dialog in den nächsten Jahren über die ehrenamtlichen Strukturen der DGB-Kreisund Stadtverbände in die Fläche tragen. Im Rahmen dieses Zukunftsdialogs wollen wir mit den Menschen diskutieren, welche Bedingungen aus ihrer Sicht vor Ort erfüllt sein müssen, damit sie ein gutes, selbstbestimmtes und sicheres Leben führen können.
Wir wollen mit dem Zukunftsdialog deutlich machen, dass Gewerkschaften als solidarische Mitmachorganisationen und mit ihrem Engagement für Gute Arbeit und ein gutes Leben jeder und jedem die Möglichkeit bieten, etwas zu verändern. Und wir wollen mit dem Zukunftsdialog einen starken Beitrag leisten, den rechtspopulistischen Spaltern unserer Gesellschaft Einhalt zu gebieten.

\section{AUTOR}

THOMAS FISCHER, Abteilungsleiter Grundsatzangelegenheiten und Gesellschaftspolitik beim DGB-Bundesvorstand. Arbeitsschwerpunkte: Demokratie und gewerkschaftliche Gesellschaftspolitik, Zukunft der Erwerbsgesellschaft, Arbeitsbeziehungen und Sozialpartnerschaft. 